\title{
SEP performance of MFSK in Rician fading channel based on MGF method
}

\author{
Dimpal joshi ${ }^{1}$, Kapil gupta ${ }^{2}$ \\ ${ }^{1}$ Mody institute of Technology and Science, Lakshmangarh, Sikar-332311(Rajasthan) \\ ${ }^{2}$ Mody institute of Technology and Science Lakshmangarh, Sikar-332311(Rajasthan)
}

\begin{abstract}
Symbol error probability (SEP) of non-coherent frequency shift keying over slow flat Rician fading channel is calculated with maximal ratio diversity combining (MRC) technique using moment generating function(MGF). We assume that channel side information is known to the receiver. Error performance plots of modulation technique has been drawn for different values of Rician factor $\mathrm{K}$, diversity order $\mathbf{N}$ and modulation order $\mathrm{M}$. The results presented here are provide information about error performance over fading channel.
\end{abstract}

Keywords: MFSK (M-ary frequency shift keying), MRC (maximal ratio combining), MGF (moment generating function), SEP (symbol error probability).

\section{INTRODUCTION}

With the advent of high data rate wireless communications, there has been a renewed interest in linear $M$-ary modulation systems, mostly due to their capability of sending multiple bits per transmitted symbol. An inherent problem found in wireless environments is often the severe multipath existing between the transmitter and receiver caused by reflections, diffraction, and refraction of the transmitter signal. The overall effect of multipath is to produce a fluctuating or fading signal at the destination receiver, which greatly degrades the quality of reception. Diversity is a technique which is based on the principle of providing multiple faded replica of same information bearing signal to the receiver [1].Several diversity techniques are used to combat the effect of fading and improve coverage capacity and reliability in wireless communication systems such as transmitter, receiver [2], space [3], frequency, polarization diversity [4]. Among all the conventional diversity combining scheme MRC is known to be optimum [5].

MFSK communication system operates in environment where large bandwidths are available but signal power is limited. In general data rate can be improved by increasing number of modulation order $\mathrm{M}$. If this is to be done without degrading probability of error then we could achieve high data rate without compromising with system performance.

\section{PERFORMANCE MEASURE}

2.1 Average Signal-to-Noise Ratio

Signal-to-noise ratio (SNR) is the most common performance measure characteristic of a digital communication system. SNR is measured at the output of the receiver and is directly related to the data detection process. The term noise in average signal-to-noise ratio refers to the ever-present thermal noise at the input to the receiver and the word average means to statistical averaging over the probability distribution of the fading[6].

$$
\bar{\gamma}=\int_{0}^{\infty} \gamma \cdot p_{\gamma}(\gamma) d \gamma
$$

Where $\gamma$ denotes the instantaneous SNR at the receiver output and $p_{\gamma}(\gamma)$ denotes the probability density function (PDF) of $\gamma$.

\subsection{Average Symbol Error Probability}

This performance criterion average symbol error probability (SEP) is the one that is most revealing about the nature of the system behaviour. The primary reason for the difficulty in evaluating average SEP lies in the fact that the conditional SEP is, in general, a nonlinear function of the instantaneous SNR, the nature of the nonlinearity being a function of the modulation/detection scheme employed by the system. The average SEP can be written as [6]

$$
\begin{aligned}
& \qquad P_{S}(E)=\int_{0}^{\infty} P_{s}\left(\frac{E}{\gamma}\right) \cdot p_{\gamma}(\gamma) d \gamma \ldots \ldots \\
& \text { Where, } P_{s}\left(\frac{E}{\gamma}\right) \text { is conditional SEP. }
\end{aligned}
$$

\section{METHOD}

3.1 Moment generating function

Moment-generating function of a random variable is an alternative representation of its probability distribution. Thus this approach provides an alternative way to compute results compared with working directly with probability density functions. There are particularly simple results for the moment-generating functions of. The MGF for a non-negative random variable $\gamma$ with distribution $p_{\gamma}(\gamma), \gamma \geq 0$, is defined as

$$
M_{\gamma}(s)=\int_{0}^{\infty} p_{\gamma}(\gamma) e^{s \gamma} d \gamma
$$

Where $p_{\gamma}(\gamma)$ denotes the probability density function (PDF) of $\gamma$.

MGF for common rician fading distribution with factor $\mathrm{K}$ and diversity order $\mathrm{N}$ is given by

$M_{\gamma}(s)=\left(\frac{N+K}{N+K-S \bar{\gamma}}\right)^{N} \cdot \exp \left(\frac{K S \bar{\gamma}}{N+K-S \bar{\gamma}}\right) \ldots$ 


\section{ERROR PROBABILITY FOR MFSK}

In an M-ary FSK scheme, the transmitted signals are defined by

$$
s_{i}(t)=\sqrt{\frac{2 E}{T}} \cos 2 \pi\left(f_{o}+(i-1) \Delta f\right) t
$$

Where $f_{0} T$ is taken as an integer.

The conditional symbol error probability of MFSK is given by [7]

$$
P_{s}\left(\frac{E}{\gamma}\right)=\frac{1}{M} \sum_{d=2}^{M}-1^{d} \cdot\left(\begin{array}{c}
M \\
d
\end{array}\right) \cdot \exp \left(-\frac{(d-1) \bar{\gamma}}{d}\right) \ldots
$$

After substitution of $P_{S}\left(\frac{E}{\gamma}\right)$ from (5) into (2) and by considering application of MGF from (3) we get

$$
P_{S}(E)=\frac{1}{M} \sum_{d=2}^{M}-1^{d} \cdot\left(\begin{array}{l}
M \\
d
\end{array}\right) \cdot M_{\gamma}\left(-\frac{d-1}{d}\right) \ldots
$$

Using MGF relation for rician fading channel from (4)

$$
\begin{aligned}
& M_{\gamma}\left(-\frac{d-1}{d}\right) \\
& =\frac{1+K}{1+K+\left(\frac{d-1}{d}\right) \bar{\gamma}} \cdot \exp \left(-\frac{K\left(\frac{d-1}{d}\right) \bar{\gamma}}{1+K+\left(\frac{d-1}{d}\right) \bar{\gamma}}\right) \cdots
\end{aligned}
$$

Finally by putting (7) into (6), the symbol error probability of coherent MDPSK over Rician fading channel with Rician parameter $\mathrm{K}$ is

$P_{S}(E)$

$=\frac{1}{M} \sum_{d=2}^{M}-1^{d} \cdot\left(\begin{array}{l}M \\ d\end{array}\right) \cdot\left(\frac{N}{N+K+\left(\frac{d-1}{d}\right) \bar{\gamma}}\right)^{N} \cdot \exp \left(-\frac{K\left(\frac{d-1}{d}\right)}{\frac{N+K}{\bar{\gamma}}+\left(\frac{d-1}{d}\right)}\right)$

The symbol error probability for coherent MDPSK over Rayleigh fading channel can be obtained by substitution of $\mathrm{K}=0$ in (8), gives

$$
P_{S}(E)=\frac{1}{M} \sum_{d=2}^{M}-1^{d} \cdot\left(\begin{array}{l}
M \\
d
\end{array}\right) \cdot\left(\frac{1}{1+\left(\frac{d-1}{d}\right) \bar{\gamma}}\right) \ldots
$$

When $\mathrm{K}$ approaches infinity, (8) reduce to (5).

\section{RESULTS AND DISCUSSION}

Here in fig 1-3 we show error performance plots of MFSK is made under Rician fading with MRC diversity combining for range of Rician fading parameter $\mathrm{K}$, diversity order $\mathrm{N}$ and modulation order $\mathrm{M}$. These curves provide information to estimate required transmitted energy to achieve certain SEP.

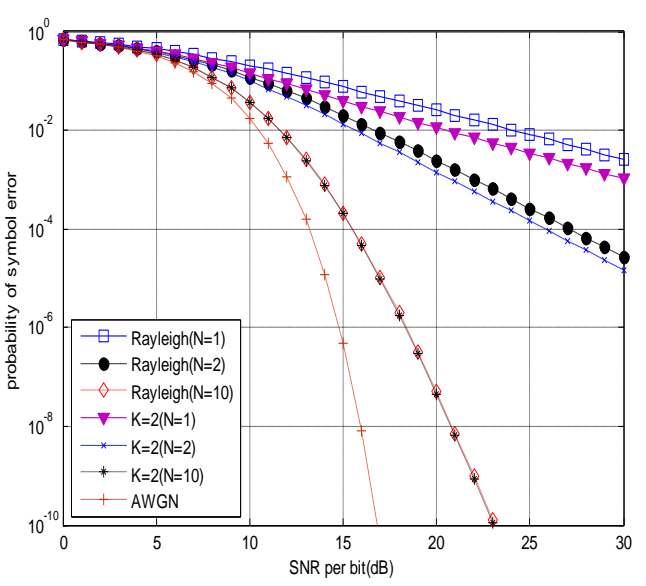

Fig.1: SEP for MFSK over Rician, Rayleigh and AWGN fading channel for different values of $K$ and $N$ and $M=8$

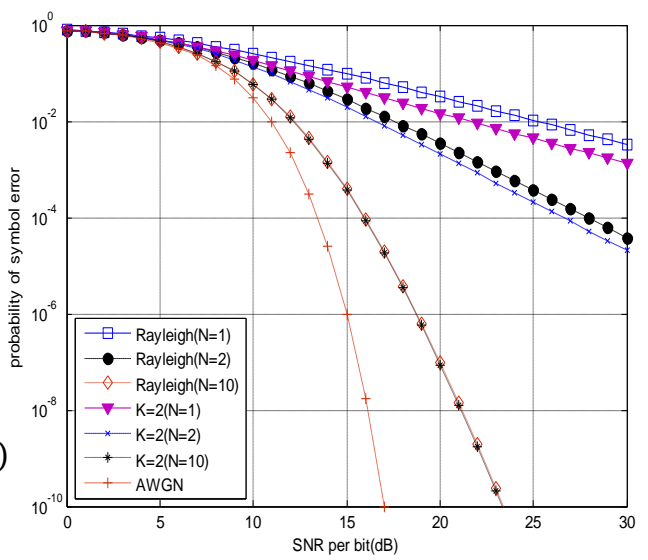

Fig.2: SEP for MFSK over Rician, Rayleigh and AWGN fading channel for different values of $K$ and $\mathrm{N}$ and $\mathrm{M}=16$

TABLE 1: SEP of MFSK for different values of $K$, $M$ and $N(S N R=20)$

\begin{tabular}{|l|l|l|}
\hline \multirow{2}{*}{$\begin{array}{c}\text { Values of } \\
\text { and }\end{array}$} & \multicolumn{2}{c|}{ symbol error probability } \\
\cline { 2 - 3 } & $\mathbf{M = 8}$ & $\mathbf{M = 1 6}$ \\
\hline $\mathrm{K}=0$ \&N=1 & 0.0253 & 0.0322 \\
\hline $\mathrm{K}=0 \& \mathrm{~N}=2$ & 0.0024 & 0.0035 \\
\hline $\mathrm{K}=0 \& \mathrm{~N}=10$ & $5.0902 \mathrm{e}-08$ & $9.8735 \mathrm{e}-08$ \\
\hline $\mathrm{K}=2 \& \mathrm{~N}=1$ & 0.0113 & 0.0146 \\
\hline $\mathrm{K}=2 \& \mathrm{~N}=2$ & 0.0014 & 0.0021 \\
\hline $\mathrm{K}=2 \& \mathrm{~N}=10$ & $4.5318 \mathrm{e}-08$ & $8.7985 \mathrm{e}-08$ \\
\hline $\mathrm{K}=$ inf & $6.7506 \mathrm{e}-22$ & $1.4466 \mathrm{e}-21$ \\
\hline
\end{tabular}




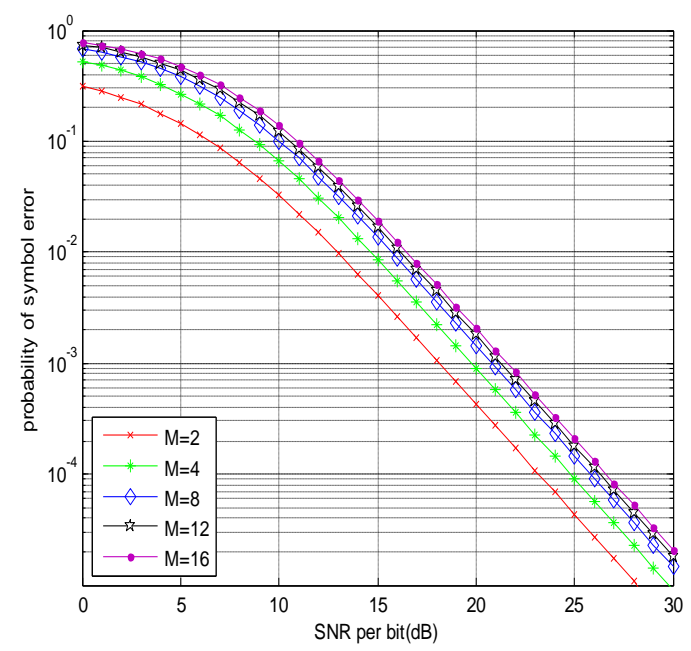

Fig.3: SEP for MFSK over Rician fading channel for different values of $M$ and $K=2, N=2$

In fig. 3 performance comparison is made with different values of modulation order M for MFSK. In this fig. five curves are plotted, which correspond to five values of modulation order $\mathrm{M}=2,4,8,12$ and 16. As expected for $\mathrm{M}=16 \mathrm{MFSK}$ has poorest performance but there is minute performance degradation for $\mathrm{M}=2$ to 16 . So we can achieve high data rate by increasing value of modulation order $\mathrm{M}$ without degrading system performance.

\section{CONCLUSION}

In this paper Error performance, based on MGF approach which is better in terms of applicability, of MFSK under Rician fading channel with MRC diversity combining are analyzed and SEP is calculated. Based on numerical calculation SEP of non-coherent MFSK is graphically plotted and compared for different values of M. MGF method performs far better because direct integration suffers from numerical instability and inaccuracy due to the presence of infinite integration limits or may contain complex mathematical functions. Many of these results can be extended for other cases of diversity reception to combat fading effects.

\section{REFERENCES}

[1] Gordon L. Stuber, "Principle of mobile communication" springer international edition.

[2] Ahmed J. Jmeel," Performance enhancement of wireless communication systems using transmit and receive diversity. 2010 7th International MultiConference on Systems, Signals and Devices, "IEEE 2010.

[3] Yihua Deng, Xiangyu Yu and Shengli Xie," A New Scheme for Jointed Space-Polarization Diversity and Its Performance Analysis," IEEE 2008.

[4] Yujiang Wu, Zaiping Nie, “ On Improving the Performance of Polarization Diversity in Wireless Communication Systems IEEE 2006.

[5] Performance analysis of OT-MRC over L.I.D nakagami and non-I.I.d Rayleigh fading channel,IEEE, vol. 55 no 6, November 2006.

[6] M.K. Simon and M.S. Alouini, "Digital Communications over fading channels".

[7] A. Goldsmith, "wireless communication". 